\title{
ON AN OPTIMIZATION PROBLEM WITH COST OF RAPID VARIATION OF CONTROL
}

\author{
D. IDCZAK, K. KIBALCZYC and S. WALCZAK ${ }^{1}$
}

(Received 14 January 1993; revised 10 March 1993)

\begin{abstract}
In the paper we give sufficient conditions for the existence of a solution for a DarbouxGoursat optimization problem with a cost functional depending on the number of switchings of a control and the rapidity of its changes. An application is given to a gas absorption problem.
\end{abstract}

\section{Introduction}

One of the basic optimization problems is the following problem: we are given a control system

$$
\begin{gathered}
\dot{x}=f(t, x, u), \quad x(0)=x_{0}, \\
u(\cdot) \in U
\end{gathered}
$$

and a cost functional

$$
I(x, u)=\int_{0}^{T} f^{0}(t, x, u) d t
$$

where $f_{0}:[0, T] \times R^{n} \times R^{m} \rightarrow R, f:[0, T] \times R^{n} \times R^{m} \rightarrow R^{n}, x(\cdot)$ is an absolutely continuous function on $[0, T], u(\cdot)$ is a measurable function and belongs to a fixed set $U$ of admissible controls.

The problem consists of the determination of the minimum of the functional $I(x, u)$ under conditions $(0.1),(0.2)$. (For details, see [3].)

In many technical questions related to automatic control, it is necessary to take account of the costs connected with the number of switchings of a control and the

'Institute of Mathematics, Łódź University, ul. Stefana Banacha 22, 90-238 Łódz, Poland

(C) Australian Mathematical Society, 1994, Serial-fee code 0334-2700/94 
speed of its changes. In a few papers $[2,4,7,8,10,11]$ the authors considered system $(0.1-0.2)$ with a cost functional of the form

$$
I(x, u)=\int_{0}^{T} f^{0}(t, x(t), u(t)) d t+\Phi(u),
$$

where $\Phi(u)$ is a functional depending on the number of switchings, the rapidity of changes or on the total variation of the control $u$.

In [8], the author gives sufficient conditions for the existence of an optimal solution to $(0.1),(0.2),(0.4)$ in the case when

$$
\Phi(u)=\gamma_{1} N(u)+\gamma_{2} S(u), \quad \gamma_{1}, \gamma_{2}>0,
$$

where $N(u)$ stands for the number of points of discontinuity of the control $u$, while $S(u)=\sup \left\{\left(\left|u(t)-u\left(t^{\prime}\right)\right|\right) /\left(\left|t-t^{\prime}\right|\right), t \neq t^{\prime}, u\right.$ is continuous on $\left.\left[t, t^{\prime}\right]\right\}$. Necessary optimality conditions for this problem are given in [4]. In the case when $\Phi(u)=\bigvee u$ (total variation of $u$ ), such a problem was considered in [7]. In [10, 11], effective methods of the numerical solving of such problems are given.

It seems that the results included in the above-mentioned papers can have practical applications (cf. MR 86b49029).

In our paper we consider an optimization problem, analogous to problem (0.1)(0.5), for systems with distributed parameters of the form

$$
\frac{\partial^{2} z}{\partial x \partial y}=f\left(x, y, z, \frac{\partial z}{\partial x}, \frac{\partial z}{\partial y}, u\right)
$$

The basic results of the paper are a theorem on the existence of Carathéodory solutions for systems of form (0.6) (cf. Section 1) and the theorem on the existence of optimal processes (Sections 2 and 3). Necessary optimality conditions for these systems will be considered in the next paper. In the last section of the paper (Section 4) we consider some chemical interpretation of the optimal control problem described by $(0.6)$.

\section{On the existence of Carathéodory solutions for Darboux-Goursat systems}

To begin with, we shall give the definition of absolutely continuous functions of several variables. In the space of these functions we shall investigate the existence of solutions of hyperbolic systems of form (0.6) with Darboux-Goursat boundary conditions.

Let $F=F(Q)$ be an additive function of the interval $Q \subset P^{2}=\left\{(x, y) \in R^{2} ; 0 \leq\right.$ $x \leq 1,0 \leq y \leq 1\}$. The function $F$ is called absolutely continuous if, for any $\varepsilon>0$, 
there exists $\delta>0$ such that $\sum_{i=1}^{n}\left|F\left(Q_{i}\right)\right|<\varepsilon$ for any system of intervals $Q_{i} \subset P^{2}$ such that $\sum_{i=1}^{n}\left|Q_{i}\right|<\delta$ and int $Q_{r} \cap$ int $Q_{s}=\emptyset$ for $r \neq s$. Let $z: P^{2} \rightarrow R$. The function of an interval defined by the formula

$$
F_{z}(Q)=z\left(x_{2}, y_{2}\right)-z\left(x_{1}, y_{2}\right)+z\left(x_{1}, y_{1}\right)-z\left(x_{2}, y_{1}\right),
$$

where $Q=\left\{(x, y) \in R^{2} ; 0 \leq x_{1} \leq x \leq x_{2} \leq 1,0 \leq y_{1} \leq y \leq y_{2} \leq 1\right\}$, is called a function associated with $z$. A function $z: P^{2} \rightarrow R$ is called an absolutely continuous function on $P^{2}$ if the associated function $F_{z}(Q)$ is an absolutely continuous function of the interval $Q$ and each of the functions $z(x, 0)$ and $z(0, y)$ is an absolutely continuous function of one variable on the interval $[0,1]$. The space of absolutely continuous functions on $P^{2}$ will be denoted by $A C\left(P^{2}, R\right)$ or, shortly, by $A C$.

It can be demonstrated (cf. [13]) that a necessary and sufficient condition for $z$ to belong to $A C\left(P^{2}, R\right)$ is the following integral representation:

$$
z(x, y)=\int_{0}^{x} \int_{0}^{y} l^{1,2}(x, y)+\int_{0}^{x} l^{1}(x)+\int_{0}^{y} l^{2}(y)+c,
$$

where $l^{1,2} \in L^{1}\left(P^{2}\right), l^{1}, l^{2} \in L^{1}([0,1]), c \in R$. Making use of (1.1), one can prove that the function $z \in A C$ possesses the partial derivatives (in the classical sense) $\partial z / \partial x=\int_{0}^{y} l^{1,2}+l^{1}, \partial z / \partial y=\int_{0}^{x} l^{1,2}+l^{2}$ and $\partial^{2} z / \partial x \partial y=l^{1,2}$ defined for $(x, y) \in P^{2}$ a.e. (cf. [14]).

In the space $A C\left(P^{2}, R\right)$, the norm is defined by the formula

$$
\|z\|_{A C}=\left\|l^{1,2}\right\|_{L_{1}\left(P^{2}\right)}+\left\|l^{1}\right\|_{L_{1}([0,1])}+\left\|l^{2}\right\|_{L_{1}([0,1])}+|c| .
$$

It is easy to see that the space $A C$ with norm (1.2) is a Banach space.

The definition of absolutely continuous functions of two variables can easily be generated by induction to the case of functions of several variables. A real function $z$ is called absolutely continuous on

$$
P^{k}=\left\{x \in R^{k}, 0 \leq x^{i} \leq 1, i=1,2, \ldots, k\right\}, \quad k \geq 2,
$$

if the associated function $F_{z}(Q)$ (cf. [6,9]) is an absolutely continuous function of the interval $Q \subset P^{k}$ and each of the functions $z\left(0, x^{2}, \ldots, x^{k}\right), \ldots, z\left(x^{1}, \ldots, x^{k-1}, 0\right)$ is an absolutely continuous function of $(k-1)$ variables. Absolutely continuous functions of $k$ variables, $k>2$, have properties analogous to those of functions of two variables (cf. [13]). In the sequel, we shall occupy ourselves with the space of functions of two variables.

Denote by $A C\left(P^{2}, R^{m}\right)$ the space of vector functions $z=\left(z^{1}, \ldots, z^{m}\right)$ where $z^{i} \in A C\left(P^{2}, R\right)$ for $i=1,2, \ldots, m$. In this space let us consider a system of equations of the form

$$
\frac{\partial^{2} z}{\partial x \partial y}=f\left(x, y, z, \frac{\partial z}{\partial x}, \frac{\partial z}{\partial y}\right)
$$


with the Darboux-Goursat boundary conditions

$$
z(x, 0)=\varphi(x), \quad z(0, y)=\psi(y),
$$

where $f=\left(f^{1}, \ldots, f^{m}\right), f: P^{2} \times\left(R^{m}\right)^{3} \rightarrow R^{m}$. We shall assume that:

$1^{0}$ the functions $\varphi$ and $\psi$ are absolutely continuous on $[0,1]$ and $\varphi(0)=$ $\psi(0)=c$,

$2^{0} \quad$ there exists a constant $L>0$ such that

$$
\begin{aligned}
\mid f^{i}\left(x, y, w_{0}, w_{1}, w_{2}\right) & -f^{i}\left(x, y, z_{0}, z_{1}, z_{2}\right) \mid \\
\leq & L\left(\left|w_{0}-z_{0}\right|+\left|w_{1}-z_{1}\right|+\left|w_{2}-z_{2}\right|\right)
\end{aligned}
$$

for $(x, y) \in P^{2}$ a.e. and any points $\left(w_{0}, w_{1}, w_{2}\right),\left(z_{0}, z_{1}, z_{2}\right)$ of the space $\left(R^{m}\right)^{3}, j=1,2, \ldots, m$,

$3^{0}$ for any point $\left(z_{0}, z_{1}, z_{2}\right) \in\left(R^{m}\right)^{3}$, the function $f\left(\cdot, \cdot, z_{0}, z_{1}, z_{2}\right)$ is measurable on $P^{2}$,

$4^{0}$ there exists $\bar{z}=\left(\bar{z}_{0}, \bar{z}_{1}, \bar{z}_{2}\right)$ such that the function $f\left(\cdot, \cdot, \bar{z}_{0}, \bar{z}_{1}, \bar{z}_{2}\right)$ is integrable on $P^{2}$.

A function $z \in A C\left(P^{2}, R^{m}\right)$ will be called a Caratheodory solution if it satisfies (1.3) for $(x, y) \in P^{2}$ a.e. and boundary conditions (1.4) for any $x, y \in[0,1]$.

We shall prove

THEOREM 1. If assumptions (1.5) are satisfied, then system of equations (1.3) with boundary conditions (1.4) possesses a unique Carathéodory solution in the space $A C\left(P^{2}, R^{m}\right)$ of absolutely continuous functions.

PROOF. It is easy to observe that, by substituting

$$
w(x, y)=z(x, y)-\varphi(x)-\psi(y)+c, \quad c=\varphi(0)=\psi(0),
$$

boundary value problem (1.3)-(1.4) can be reduced to a problem with homogeneous boundary conditions. Therefore, without loss of generality, one may assume that $\varphi(x) \equiv 0$ and $\psi(y) \equiv 0$. It follows from (1.1) that any function $z \in A C\left(P^{2}, R^{m}\right)$ satisfying the conditions $z(x, 0) \equiv 0$ and $z(0, y) \equiv 0$ can be represented in the form

$$
z(x, y)=\int_{0}^{x} \int_{0}^{y} g(x, y) d x d y,
$$

where $g \in L^{1}\left(P^{2}, R^{m}\right)$. So, (1.3) with homogeneous boundary conditions can be represented in the form

$$
g(x, y)=f\left(x, y, \int_{0}^{x} \int_{0}^{y} g, \int_{0}^{y} g, \int_{0}^{x} g\right),
$$

where $g$ satisfies (1.6). 
Let $k>1$ be a number satisfying the inequality

$$
2 m L\left(\frac{1}{k^{2}}+\frac{1}{k}\right)=\alpha<1,
$$

where $L$ is the constant from the Lipschitz condition (cf. (1.5)). In the space $L^{1}\left(P^{2}, R^{m}\right)$ let us introduce a Bielecki norm (cf. [1]) defined by the formula

$$
\|g\|_{k}=\int_{0}^{1} \int_{0}^{1} e^{-k(x+y)}|g(x, y)| d x d y .
$$

It is easy to show that

$$
e^{-2 k}\|g\|_{L^{1}} \leq\|g\|_{k} \leq\|g\|_{L^{1}} .
$$

Consequently, the norms $\|g\|_{k}$ and $\|g\|_{L^{1}}$ are equivalent.

Denote by $F=\left(F^{1}, \ldots, F^{m}\right): L^{1}\left(P^{2}, R^{m}\right) \rightarrow L^{1}\left(P^{2}, R^{m}\right)$ an operator defined by the formula

$$
F^{j}(g)(x, y)=f^{j}\left(x, y, \int_{0}^{x} \int_{0}^{y} g(x, y), \int_{0}^{y} g(x, y), \int_{0}^{x} g(x, y)\right) .
$$

It follows from assumptions (1.5) that the operator $F$ is well-defined. We shall demonstrate that $F$ is a contracting operator if the norm in the space $L^{1}\left(P^{2}, R^{m}\right)$ is defined by (1.9). We have

$$
\begin{aligned}
& \|F(g)-F(h)\|_{k}= \\
& \int_{0}^{1} \int_{0}^{1} e^{-k(x+y)}\left|f\left(x, y, \int_{0}^{x} \int_{0}^{y} g, \int_{0}^{y} g, \int_{0}^{x} g\right)-f\left(x, y, \int_{0}^{x} \int_{0}^{y} h, \int_{0}^{y} h, \int_{0}^{x} h\right)\right| d x d y \\
& \leq m L \int_{0}^{1} \int_{0}^{1} e^{-k(x+y)} \int_{0}^{x} \int_{0}^{y}|g-h|+m L \int_{0}^{1} \int_{0}^{1} e^{-k(x+y)} \int_{0}^{y}|g-h| \\
& +m L \int_{0}^{1} \int_{0}^{1} e^{-k(x+y)} \int_{0}^{x}|g-h| .
\end{aligned}
$$

Integrating the last integral by parts, we obtain

$$
\begin{aligned}
\int_{0}^{1} & \int_{0}^{1}\left(e^{-k(x+y)} \int_{0}^{x}|g-h|(s, y) d s\right) d x d y \\
& =\int_{0}^{1}\left[\int_{0}^{1}\left(e^{-k(x+y)} \int_{0}^{x}|g-h|(s, y) d s\right) d x\right] d y \\
& =\int_{0}^{1}\left[-\left.\frac{1}{k} e^{-k(x+y)} \int_{0}^{x}|g-h|(s, y) d s\right|_{x=0} ^{x=1}+\int_{0}^{1} \frac{1}{k} e^{-k(x+y)}|g-h|(x, y) d x\right] d y \\
& =\int_{0}^{1} \int_{0}^{1}\left(-\frac{1}{k} e^{-k(1+y)}+\frac{1}{k} e^{-k(x+y)}\right)|g-h|(x, y) d x d y
\end{aligned}
$$




$$
\begin{aligned}
& =\frac{1}{k} \int_{0}^{1} \int_{0}^{1}\left(-e^{-k(1-x)}+1\right) e^{-k(x+y)}|g-h|(x, y) d x d y \\
& \leq \frac{1}{k}\|g-h\|_{k} .
\end{aligned}
$$

In an analogous way we shall obtain the inequality

$$
\int_{0}^{1} \int_{0}^{1} e^{-k(x+y)} \int_{0}^{y}|g-h|(x, y) \leq \frac{1}{k}\|g-h\|_{k} .
$$

Integrating by parts twice, we get the estimate

$$
\begin{aligned}
& \int_{0}^{1} \int_{0}^{1} e^{-k(x+y)} \int_{0}^{x} \int_{0}^{y}|g-h|(x, y) \\
& \quad=\frac{1}{k^{2}} \int_{0}^{1} \int_{0}^{1}\left[e^{-k(2-x-y)}-e^{-k(1-x)}-e^{-k(1-y)}+1\right] e^{-k(x+y)}|g-h|(x, y) d x d y \\
& \leq \frac{2}{k^{2}}\|g-h\|_{k} .
\end{aligned}
$$

Consequently, from (1.12) we obtain

$$
\|F(g)-F(h)\|_{k} \leq 2 m L\left(\frac{1}{k^{2}}+\frac{1}{k}\right)\|g-h\|_{k}=\alpha\|g-h\|_{k} .
$$

Since the number $k$ satisfies (1.8), we ascertain that $F$ (cf. (1.11)) is a contracting operator. So, there exists exactly one point $g_{0} \in L^{1}\left(P^{2}, R^{m}\right)$ such that $g_{0}=F\left(g_{0}\right)$. Adopting

$$
z_{0}(x, y)=\int_{0}^{x} \int_{0}^{y} g_{0}(x, y),
$$

we obtain a solution of (1.3) in the space of absolutely continuous functions on $P^{2}$.

\section{On the existence of optimal solutions for some control problem}

We shall first prove a theorem on the continuous dependence of solutions of (1.3) upon the function parameter (control). We shall make use of the following:

LEMMA 1. (cf. [5]) If a function $\varphi: P^{2} \times R^{l} \rightarrow R, l \geq 1$, satisfies the conditions:

$1^{0} \varphi(\cdot, \cdot, v)$ is measurable on $P^{2}$ with any $v \in R^{\prime}$,

$2^{0} \varphi(x, y, \cdot)$ is continuous on $R^{2}$ with any $(x, y) \in P^{2}$,

$3^{0}$ for any $u(\cdot, \cdot) \in L^{p_{1}}\left(P^{2}, R^{l}\right), 1 \leq p_{1} \leq \infty$, the function $\varphi(\cdot, \cdot, u(\cdot, \cdot)) \in L^{p_{2}}\left(P^{2}, R\right), 1 \leq p_{2}<\infty$,

then the operator $\Phi: L^{p_{1}}\left(P^{2}, R\right) \rightarrow L^{p_{2}}\left(P^{2}, R\right)$ defined by the formula $\Phi(u)(x, y)=$ $\varphi(x, y, u(x, y))$ is continuous. 
Let us now consider a control system

$$
\frac{\partial^{2} z}{\partial x \partial y}=f\left(x, y, z, \frac{\partial z}{\partial x}, \frac{\partial z}{\partial y}, u\right), \quad z(x, 0)=0, \quad z(0, y)=0,
$$

where $z \in A C\left(P^{2}, R^{m}\right), u \in L^{s}\left(P^{2}, R^{r}\right), f=\left(f^{1}, \ldots, f^{m}\right), 1 \leq s \leq \infty, r \geq 1$. We shall assume that:

1. there exists a constant $L>0$ such that

$$
\begin{aligned}
\left|f^{j}\left(x, y, w_{0}, w_{1}, w_{2}, u\right)-f^{j}\left(x, y, z_{0}, z_{1}, z_{2}, u\right)\right| \\
\leq L\left(\left|w_{0}-z_{0}\right|+\left|w_{1}-z_{1}\right|+\left|w_{2}-z_{2}\right|\right)
\end{aligned}
$$

for any $(x, y) \in P^{2}, w_{i}, z_{i} \in R^{m}, i=0,1,2, u \in R^{r}$,

2. for any $z_{i} \in R^{m}$ and $u \in R^{r}$, the function $f\left(\cdot, \cdot, z_{0}, z_{1}, z_{2}, u\right)$ is measurable on $P^{2}$, for any $(x, y) \in P^{2}$ and $z_{i} \in R^{m}$, the function $f\left(x, y, z_{0}, z_{1}, z_{2}, \cdot\right)$ is continuous on $R^{r}$,

3. for any control $u \in L^{s}\left(P^{2}, R^{r}\right)$, there exists a point $\bar{z}_{i} \in R^{m}, i=$ $0,1,2$, such that the function $f^{j}\left(x, y, \bar{z}_{0}, \bar{z}_{1}, \bar{z}_{2}, u(x, y)\right)$ is integrable on $P^{2}, j=1,2, \ldots, m$.

From the above assumptions as well as from Theorem 1, it follows that, for any control $u \in L^{s}\left(P^{2}, R^{r}\right)$, there exists exactly one solution of (2.1) in the space of absolutely continuous functions $A C\left(P^{2}, R^{m}\right)$. This solution will be denoted by $z_{u}$ and called the trajectory of (2.1) corresponding to the control $u$. Since $z_{u}(x, 0)=0$ and $z_{u}(0, y)=0$, therefore the norm of the trajectory $z_{u}$ is defined by the formula (cf. (1.2))

$$
\left\|z_{u}\right\|=\int_{P^{2}} \int\left|\frac{\partial^{2} z_{u}}{\partial x \partial y}(x, y)\right| d x d y .
$$

We shall prove

THEOREM 2. If assumptions (2.2) are satisfied and the sequence of controls $u_{n}$ tends to $u_{0}$ in the space $L^{s}\left(P^{2}, R^{r}\right)$, then the sequence of trajectories $z_{u_{n}}$ tends to $z_{u_{0}}$ in the space $A C\left(P^{2}, R^{m}\right)$.

PROOF. Denote by $F_{u_{n}}=\left(F_{u_{n}}^{1}, \ldots, F_{u_{n}}^{m}\right): L^{1}\left(P^{2}, R^{m}\right) \rightarrow L^{1}\left(P^{2}, R^{m}\right)$ an operator defined by the formula

$$
F_{u_{n}}^{j}(g)(x, y)=f^{j}\left(x, y, \int_{0}^{x} \int_{0}^{y} g, \int_{0}^{y} g, \int_{0}^{x} g, u_{n}(x, y)\right)
$$

where $j=1,2, \ldots, m ; g \in L^{1}\left(P^{2}, R^{m}\right),(x, y) \in P^{2}$. Identically as in the proof of Theorem 1, we can show that there exists $k>1$ such that $\alpha=2 m L\left(\frac{1}{k^{2}}+\frac{1}{k}\right)<1$ and

$$
\left\|F_{u_{n}}(g)-F_{u_{n}}(h)\right\|_{k} \leq \alpha\|g-h\|_{k}
$$


for any $g, h \in L^{1}\left(P^{2}, R^{m}\right)$ and $n=0,1,2, \ldots$, where $\|\cdot\|_{k}$ stands for the norm defined by (1.9).

For any control $u_{n}$, the operator $F_{u_{n}}$ possesses a fixed point $g_{n}=F_{u_{n}}\left(g_{n}\right)$ such that

$$
z_{u_{n}}(x, y)=\int_{0}^{x} \int_{0}^{y} g_{n}(x, y) d x d y, \quad n=0,1,2, \ldots,
$$

and $z_{u_{n}}$ is a trajectory of system (2.1). Inequality (2.4) implies

$$
\begin{aligned}
\left\|g_{n}-g_{0}\right\|_{k} & =\left\|F_{u_{n}}\left(g_{n}\right)-F_{u_{0}}\left(g_{0}\right)\right\|_{k} \\
& \leq\left\|F_{u_{n}}\left(g_{n}\right)-\mid F_{u_{n}}\left(g_{0}\right)\right\|_{k}+\left\|F_{u_{n}}\left(g_{0}\right)-F_{u_{0}}\left(g_{0}\right)\right\|_{k} \\
& \leq \alpha\left\|g_{n}-g_{0}\right\|_{k}+\left\|F_{u_{n}}\left(g_{0}\right)-F_{u_{0}}\left(g_{0}\right)\right\|_{k} .
\end{aligned}
$$

Making use of (1.10), we get

$$
\left\|g_{n}-g_{0}\right\|_{L^{1}} \leq \frac{e^{2 k}}{1-\alpha}\left\|F_{u_{n}}\left(g_{0}\right)-F_{u_{0}}\left(g_{0}\right)\right\|_{L^{1}}
$$

Since $z_{u}(x, 0) \equiv 0$ and $z_{u}(0, y) \equiv 0$, we have (cf. (2.3))

$$
\left\|z_{u_{n}}-z_{u_{0}}\right\|_{A C\left(P^{2}, R^{m}\right)}=\left\|g_{n}-g_{0}\right\|_{L^{1}\left(P^{2}, R^{m}\right)} .
$$

Consequently,

$$
\left\|z_{u_{n}}-z_{u_{0}}\right\| \leq \frac{e^{2 k}}{1-\alpha}\left\|F_{u_{n}}\left(g_{0}\right)-F_{u_{0}}\left(g_{0}\right)\right\|, \quad n=1,2, \ldots
$$

From Lemma 1 it follows that the operator $F_{u}$ is continuous, thus $z_{u_{n}} \stackrel{A C}{\rightarrow} z_{u_{0}}$ as $u_{n} \stackrel{L^{s}}{\rightarrow} u_{0}, 1 \leq s \leq \infty$.

A function $u_{0}: P^{2} \rightarrow R^{r_{0}}$ will be called piecewise continuous on $P^{2}$ if there exist points $0=x_{0}<x_{1}<\cdots<x_{p_{0}}=1$ and $0=y_{0}<y_{1}<\cdots<y_{q_{0}}=1$ such that the function $u_{0}$ is continuous on each interval $P_{i k}=\left(x_{i}, x_{i+1}\right) \times\left(y_{i}, y_{i+1}\right)$, $i=0,1, \ldots, p_{0}-1 ; k=0,1, \ldots, q_{0}-1$.

Let $u_{0}=\left(u_{0}^{1}, \ldots, u_{0}^{r_{0}}\right)$ be a piecewise continuous function on $P^{2}$ such that $u_{0}(x, y) \in M_{0} \subset R^{r_{0}}$ and $S_{0}\left(u_{0}\right)<\infty$, where $M_{0}$ is a compact set and the functional $S_{0}\left(u_{0}\right)$ is defined by the formula

$$
\begin{gathered}
S_{0}\left(u_{0}\right)=\sup \left\{\frac{\left|u_{0}\left(x^{\prime}, y^{\prime}\right)-u_{0}(x, y)\right|}{\left|\left(x^{\prime}, y^{\prime}\right)-(x, y)\right|}, \quad\left(x^{\prime}, y^{\prime}\right) \neq(x, y),\left(x^{\prime}, y^{\prime}\right),(x, y) \in P_{i k},\right. \\
\left.i=0,1, \ldots, p_{0}-1 ; k=0,1, \ldots, q_{0}-1\right\}
\end{gathered}
$$


The set of such functions will be denoted by $U_{0}$. Denote by $U_{1}$ the set of functions $u_{1}=u_{1}(x)=\left(u_{1}^{1}(x), \ldots, u_{1}^{r_{1}}(x)\right)$ piecewise continuous on $[0,1]$ and such that $u_{1}(x) \in M_{1}, S_{1}\left(u_{1}\right)<\infty$, where $M_{1} \subset R^{r_{1}}$ is a compact set, the functional $S_{1}\left(u_{1}\right)$ is defined by the formula

$$
S_{1}\left(u_{1}\right)=\sup \left\{\frac{\left|u_{1}\left(x^{\prime}\right)-u_{1}(x)\right|}{\left|x^{\prime}-x\right|}, \quad x^{\prime} \neq x, \quad x^{\prime}, x \in P_{i}\right\}
$$

where $P_{i}, i=1,2, \ldots, p$, denote the intervals of continuity of the function $u_{1}$.

Let $U_{2}$ stand for the set of functions $u_{2}=u_{2}(y)=u_{0}=\left(u_{2}^{1}(y), \ldots, u_{2}^{r_{2}}(y)\right)$ piecewise continuous on $[0,1]$ and such that $u_{2}(y) \in M_{2}, S_{2}\left(u_{2}\right)<\infty$, where $M_{2} \subset R^{r_{2}}$ is a compact set, the functional $S_{2}\left(u_{2}\right)$ is defined by the formula

$$
S_{2}\left(u_{2}\right)=\sup \left\{\frac{\left|u_{2}\left(y^{\prime}\right)-u_{2}(y)\right|}{\left|y^{\prime}-y\right|}, \quad y^{\prime} \neq y, \quad y^{\prime}, y \in Q_{k}\right\}
$$

and $Q_{k}, k=1,2, \ldots, q$, denote the intervals of continuity of the function $u_{2}$.

The set $U=U_{0} \times U_{1} \times U_{2}$ will be called a set of admissible controls, and its elements - admissible controls. The control $u_{0}=u_{0}(x, y)$ will be called an interval control, whereas $u_{1}=u_{1}(x)$ and $u_{2}=u_{2}(y)$ - boundary controls.

In the class of admissible controls $u=\left(u_{0}, u_{1}, u_{2}\right) \in U$, defined above, let us consider a control system of the form

$$
\begin{array}{r}
\frac{\partial^{2} z}{\partial x \partial y}=f\left(x, y, z, \frac{\partial z}{\partial x}, \frac{\partial z}{\partial y}, u\right), \quad z(x, 0)=0, \quad z(0, y)=0 \\
u(x, y) \in M=M_{0} \times M_{1} \times M_{2}, \quad z \in A C\left(P^{2}, R^{m}\right)
\end{array}
$$

with a cost functional

$$
I(z, u)=\int_{P^{2}} f^{0}(x, y, z(x, y), u(x, y)) d x d y+\sum_{i=0}^{2} \alpha_{i} N_{i}\left(u_{i}\right)+\beta_{i} S_{i}\left(u_{i}\right) \rightarrow \min
$$

where $\alpha_{i}, \beta_{i}>0, N_{i}\left(u_{i}\right)$ stands for the number of intervals of continuity of the controls $u_{i}, i=0,1,2, ; S_{i}\left(u_{i}\right)$ are defined by $(2.5)-(2.7)$.

The functionals $N_{i}$ and $S_{i}, i=0,1,2$, can be interpreted as costs connected with the number of switchings of the control $u$ and the speed of its changes, whereas the numbers $\alpha_{i}, \beta_{i}$ as weights connected with these costs. 
In the sequel, we shall assume that:

a) the function $f$ satisfies (2.2),

b) $f^{0}(\cdot, \cdot, z, u)$ is measurable with any $z \in R^{m}$ and $u \in R^{r}$, $r=r_{0}+r_{1}+r_{2} ; f^{0}(x, y, \cdot, \cdot)$ is continuous for $(x, y) \in P^{2}$,

c) $f^{0}(\cdot, \cdot, z(\cdot, \cdot), u(\cdot, \cdot))$ is an integrable function for any admissible pair $(z, u)$ where $u \in U, z=z_{u}$ is the solution of (2.8) corresponding to the control $u$,

d) there exists a constant $A$ such that

$$
\int_{P^{2}} f^{0}(x, y, z(x, y), u(x, y)) d x d y \geq A
$$

for any admissible control $u \in U$ and the trajectory $z=z_{u}$ satisfying (2.8).

Let $C \geq 1$ be a fixed real number. Denote by $U_{0}^{C}$ the set of functions

$$
U_{0}^{C}=\left\{u \in U_{0} ; N_{0}(u) \leq C \text { and } S_{0}(u) \leq C\right\} .
$$

We shall show

LEMMA 2. From each sequence $\left\{u^{n}\right\} \subset U_{0}^{c}$ one can choose a subsequence $\left\{u^{n_{k}}\right\}$ consisting of functions which possess exactly $s \leq C$ intervals of continuity. This subsequence converges almost everywhere on $P^{2}$ to a function $u^{0} \in U_{0}^{C}$, and the number of intervals of continuity of the function $u^{0}$ is equal to $s^{0} \leq s \leq C$. Moreover, for each $\varepsilon>0$, there exists $N=N(\varepsilon)$ such that $S_{0}\left(u^{0}\right) \leq S_{0}\left(u^{n_{k}}\right)+\varepsilon$ for any $n^{k} \geq N$.

Proof. Let $\left\{u^{n}\right\} \subset U_{0}^{C}$ be an arbitrary sequence. It follows from the definition of the set $U_{0}^{C}$ that the number of intervals of continuity of any function $u^{n}$ is less than $C$. So, there exist points $0=x_{0}^{n}<x_{1}^{n}<\cdots<x_{p}^{n}=1,0=y_{0}^{n}<y_{1}^{n}<\cdots<y_{q}^{n}=1$ such that the function $u^{n}$ is continuous on the intervals

$$
\begin{aligned}
P_{i k}^{n}=\left(x_{i}^{n}, x_{i+1}^{n}\right) \times\left(y_{i}^{n}, y_{i+1}^{n}\right), & \\
i & =0,1, \ldots, p-1 ; k=0,1, \ldots, q-1, p \leq C, q \leq C .
\end{aligned}
$$

(In the case $C=1$, all the functions $u^{n}$ are continuous on $P^{2}$.) Choosing a subsequence, if necessary, we may assume that the numbers $p$ and $q$ are constant for all $n=1,2, \ldots$. Put $s=p \cdot q$. From each sequence $\left\{x_{i}^{n}\right\}$ and $\left\{y_{k}^{n}\right\}$ we can choose convergent subsequences. (In the sequel, subsequences will be denoted by the same symbols as original sequences.)

Let $x_{i}^{n} \rightarrow x_{i}^{n}$ and $y_{k}^{n} \rightarrow y_{k}^{0}, i=0,1, \ldots, p ; k=0,1, \ldots, q$. We have $0=$ $x_{0}^{0} \leq x_{1}^{0} \leq \cdots \leq x_{p}^{0}=1$ and $0=y_{0}^{0} \leq y_{1}^{0} \leq \cdots \leq y_{q}^{0}=1$. Assume that, with fixed $i$ and $k, x_{i}^{0}<x_{i+1}^{0}$ and $y_{k}^{0}<y_{k+1}^{0}$. Let $\varepsilon>0$ be a sufficiently small number. Note that on the interval $P_{i k}^{0, \varepsilon}=\left(x_{i}^{0}+\varepsilon, x_{i+1}^{0}-\varepsilon\right) \times\left(y_{k}^{0}+\varepsilon, y_{k+1}^{0}-\varepsilon\right)$ 
the sequence $u^{n}$ will be compact in the topology of uniform convergence. Indeed, on this interval all the functions $u^{n}$ with a sufficiently large $n$ are defined, commonly bounded $\left(u^{n}(x, y) \in M_{0}\right)$ and equicontinuous $\left(S_{0}\left(u^{n}\right) \leq C\right)$. Consequently, from the Ascoli-Arzelà theorem it follows that there exists a uniformly convergent subsequence. Passing with $\varepsilon \rightarrow 0$, we shall obtain pointwise convergence on the whole interval $P_{i k}^{0}=\left(x_{i}^{0}, x_{i+1}^{0}\right) \times\left(y_{k}^{0}, y_{k+1}^{0}\right)$. Proceeding in this way with the successive intervals $P_{i k}^{0}$, we shall get a subsequence of $\left\{u^{n}\right\}$ converging almost everywhere to some function $u^{0} \in U_{0}^{C}$. The number of intervals of continuity of the function $u^{0}$ is equal to $s_{0} \leq s$. In the case when $x_{i}^{0}=x_{i+1}^{0}$ or $y_{k}^{0}=y_{k+1}^{0}, P_{i k}^{0}$ is a degenerate interval and its two-dimensional measure is equal to zero. Such a case may therefore be omitted.

We shall prove the second part of the proposition. Let $\varepsilon>0$ be an arbitrary number. Directly from the definition of the supremum and from (2.5) it follows that

$$
\begin{aligned}
S_{0}\left(u^{0}\right) & =\sup \left\{\frac{\left|u^{0}\left(x^{\prime}, y^{\prime}\right)-u^{0}(x, y)\right|}{\left|\left(x^{\prime}, y^{\prime}\right)-(x, y)\right|}, \quad\left(x^{\prime}, y^{\prime}\right) \neq(x, y),\left(x^{\prime}, y^{\prime}\right),(x, y) \in P_{i k}^{0}\right\} \\
& \leq \frac{\left|u^{0}\left(\bar{x}^{\prime}, \bar{y}^{\prime}\right)-u^{0}(\bar{x}, \bar{y})\right|}{\left|\left(\bar{x}^{\prime}, \bar{y}^{\prime}\right)-(\bar{x}, \bar{y})\right|}+\frac{\varepsilon}{2},
\end{aligned}
$$

where $\left(\bar{x}^{\prime}, \bar{y}^{\prime}\right)$ and $(\bar{x}, \bar{y})$ are some points of the interval $P_{i k}^{0}$. Since the sequence $\left\{u^{n_{k}}\right\}$ converges to $u_{0}$, therefore

$$
S_{0}\left(u^{0}\right) \leq \frac{\left|u^{n_{k}}\left(\bar{x}^{\prime}, \bar{y}^{\prime}\right)-u^{n_{k}}(\bar{x}, \bar{y})\right|}{\left|\left(\bar{x}^{\prime}, \bar{y}^{\prime}\right)-(\bar{x}, \bar{y})\right|}+\varepsilon
$$

for $n_{k}$ sufficiently large. Hence

$$
S_{0}\left(u^{0}\right) \leq \sup \left\{\frac{\left|u^{n_{k}}\left(x^{\prime}, y^{\prime}\right)-u^{n_{k}}(x, y)\right|}{\left|\left(x^{\prime}, y^{\prime}\right)-(x, y)\right|}\right\}+\varepsilon \leq S_{0}\left(u^{n_{k}}\right)+\varepsilon .
$$

Let us denote by $U_{1}^{C}$ and $U_{2}^{C}$ the sets

$$
\begin{array}{ll}
U_{1}^{C}=\left\{u \in U_{1} ;\right. & \left.N_{1}(u) \leq C \text { and } S_{1}(u) \leq C\right\}, \\
U_{2}^{C}=\left\{u \in U_{2} ;\right. & \left.N_{2}(u) \leq C \text { and } S_{2}(u) \leq C\right\} .
\end{array}
$$

Analogously to Lemma 2 one can show

LEMMA 3. From each sequence $\left\{u^{n}\right\} \subset U_{1}^{C}$ one can choose a subsequence $\left[u^{n_{k}}\right\}$ consisting of functions which possess exactly $s \leq C$ intervals of continuity. This subsequence converges almost everywhere on $[0,1]$ to a function $u^{0} \in U_{1}^{C}$, and the number of intervals of continuity of the function $u^{0}$ is equal to $s^{0} \leq s \leq C$. Moreover, for each $\varepsilon>0$, there exists $N=N(\varepsilon)$ such that $S_{1}\left(u^{0}\right) \leq S_{1}\left(u^{n_{k}}\right)+\varepsilon$ for any $n_{k} \geq N$. Analogously - for the set $U_{2}^{C}$. 
On the basis of the above lemmas and Theorem 2, we shall prove

THEOREM 3. If (2.10) are satisfied, then control system (2.8) with cost functional (2.9) possesses an optimal solution in the class of admissible controls $U=U_{0} \times U_{1} \times U_{2}$ and in the class of absolutely continuous trajectories $z \in A C\left(P^{2}, R^{m}\right)$.

Proof. Denote by $Z$ the set of admissible processes $(z, u)$ in $(2.8)-(2.9)$, i.e. $u \in U$ and $z$ is the trajectory of (2.8) corresponding to the control $u$. It follows from (2.10.d) that $\inf \{I(z, u) ;(z, u) \in Z\}=m>-\infty$. Let $\left\{\left(z^{n}, u^{n}\right)\right\} \subset Z$ be a minimizing sequence, i.e. $I\left(z^{n}, u^{n}\right) \rightarrow m$. It can easily be noticed that there exists $C \geq 1$ such that

$$
\sum_{i=0}^{2} \alpha_{i} N_{i}\left(u_{i}^{n}\right)+\beta_{i} S_{i}\left(u_{i}^{n}\right) \leq C .
$$

Since $\alpha_{i}, \beta_{i}>0$, from the sequence $\left\{u^{n}\right\}$ we can choose a subsequence $\left\{u^{n_{k}}\right\}$ satisfying the conditions of Lemmas 2 and 3. Let $u^{n_{k}} \rightarrow u^{0}$. From Theorem 2 we infer that

$$
\int_{P^{2}} f^{0}\left(x, y, z^{n_{k}}, u^{n_{k}}\right) \rightarrow \int_{P^{2}} f^{0}\left(x, y, z^{0}, u^{0}\right),
$$

where $z^{0}$ is the trajectory corresponding to the control $u^{0}$.

Let $\varepsilon>0$ be an arbitrary real number. It follows from (2.11) and from Lemmas 2 and 3 that

$$
m \leq I\left(z^{0}, u^{0}\right) \leq I\left(z^{n_{k}}, u^{n_{k}}\right)+\varepsilon
$$

for $n_{k}$ sufficiently large. Since $I\left(z^{n_{k}}, u^{n_{k}}\right) \rightarrow m$, from (2.12) we obtain $I\left(z^{0}, u^{0}\right)=m$.

REMARK 1 . We have assumed that $\alpha_{i}, \beta_{i}>0, i=0,1,2$. Directly from the proof of Theorem 3 it follows that, in the case when $f^{0}$ and $f$ do not depend on $u_{i}$, one can put $\alpha_{i}=0$ and $\beta_{i}=0$ for $i=0,1$ or 2 and Theorem 3 will still remain true.

REMARK 2. In the existence theorems for the classical problems of Bolza or Lagrange one assumes the convexity of the function $f^{0}$ and the convexity of the set of trajectories (cf. [3]). In Theorem 3 we managed to omit the assumptions of convexity because the cost functional (2.9) contains components depending on the number of intervals of continuity of the control $u$ and the rapidity of its changes.

\section{An optimal control problem with the bounded number of switchings of controls}

In this section we shall consider the optimal control systems with a commonly bounded number of swithchings of controls. 
Let $K_{0}, K_{1}, K_{2}$ be some fixed positive integers. Denote by $U_{K_{0}}$ a subset of $U_{0}$ (cf. (2.5)) consisting of controls $u$ whose number of continuity intervals is not greater than $K_{0}$. In an analogous way we define the sets $U_{K_{1}} \subset U_{1}$ and $U_{K_{2}} \subset U_{2}$ (cf. (2.6) and (2.7)). Let $U_{K}=U_{K_{0}} \times U_{K_{1}} \times U_{K_{2}}$.

In the set of admissible controls $u=\left(u_{0}, u_{1}, u_{2}\right) \in U_{K}$, let us consider a control system of the form

$$
\begin{aligned}
\frac{\partial^{2} z}{\partial x \partial y}= & f\left(x, y, z, \frac{\partial z}{\partial x}, \frac{\partial z}{\partial y}, u\right), \\
& z(x, 0)=0, \quad z(0, y)=0, u \in U_{K}, \quad z \in A C\left(P^{2}, R^{m}\right),
\end{aligned}
$$

with a performance index

$$
I(z, u)=\int_{P^{2}} f^{0}(x, y, z, u) d x d y+\sum_{i=0}^{2} \beta_{i} S_{i}\left(u_{i}\right),
$$

where $\beta_{i}>0$ and the functionals $S_{i}$ are defined by (2.5)-(2.7).

Now, we prove the following existence theorem for the optimal control problem (3.1)-(3.2).

THEOREM 4. If the functions $f$ and $f^{0}$ satisfy (2.2) and (2.10), then control system (3.1) with performance index (3.2) possesses an optimal solution in the class of admissible controls $u \in U_{K}$ and in the class of absolutely continuous trajectories $z \in A C\left(P^{2}, R^{m}\right)$.

PROOF. Let $Z_{k}$ denote the set of admissible processes $(z, u)$ in (3.1)-(3.2). It follows from (2.10d) that $\inf \left\{I(z, u) ;(z, u) \in Z_{k}\right\}=m>-\infty$. Let $\left\{\left(z^{n}, u^{n}\right)\right\} \subset Z_{k}$ be a minimizing sequence, i.e. $I\left(z^{n}, u^{n}\right) \rightarrow m$. Since (cf. (2.10))

$$
\int_{P^{2}} f^{0}\left(x, y, z^{n}, u^{n}\right) \geq A, \quad n=1,2, \ldots
$$

for some $A$, there exists a constant $D>0$ such that $\sum_{i=0}^{2} \beta_{i} S_{i}\left(u_{i}^{n}\right) \leq D$. By the definition of admissible controls, the number of continuity intervals of $u_{i}^{n}, n=0,1,2$, is commonly bounded. It follows from Lemmas 2 and 3 that there exists some subsequence $\left\{u^{n_{k}}\right\} \subset\left\{u^{n}\right\}$ such that $u^{n_{k}} \rightarrow u^{0}$ a.e. and $u^{0} \in U_{k}$. Further, by an identical argumentation as in the proof of Theorem 3, we obtain the assertion of Theorem 4.

REMARK 3. It is easy to notice that, in the case when functions $f^{0}$ and $f$ do not depend on $u_{i}$, we can put $\beta_{i}=0$ in (3.2), and Theorem 4 will be true, $i=0,1$ or 2 . 


\section{Application to the optimization of some chemical processes}

Consider a gas filter made in the form of a pipe filled up with a substance $S$ which absorbs a poison gas. Through the filter a mixture of air and gas is pressed at a speed $v=v(t)>a>0$ with the aid of an aggregation $A$. Denote by $\bar{u}=\bar{u}(x, t)$ the quantity of the poison gas being present in the capacity unit of the substance $S$ at a distance $x$ from the inlet of the filter and at a moment $t$. Assume the speed $v=v(t)$ to be so great that the diffusion process plays no essential role in the motion of the gas. In this case, the process of the absorption of the poison gas by the filter filled up with the substance $S$ is described by a differential equation of the form

$$
\bar{u}_{x t}(x, t)+\frac{\beta}{v(t)} \bar{u}_{t}(x, t)+\beta \gamma \bar{u}_{x}(x, t)=0
$$

under the boundary conditions

$$
\begin{aligned}
& \bar{u}(x, 0)=\bar{u}_{0} \exp \left(\frac{-\beta}{v_{0}} x\right), \\
& \bar{u}(0, t)=\bar{u}_{0},
\end{aligned}
$$

where $\bar{u}_{0}$ is the gas concentration at the inlet to the filter $\left(\bar{u}_{0}=\right.$ const. $), v(t)$ denotes the speed of the flow of the mixture of air and gas through the filter at the moment $t$, $v_{0}=v(0), \beta$ and $\gamma$ are physical quantities characterizing the given gas (for details, see [12], chapter II).

With the aid of the aggregation $A$ we are able to control the flow speed $v=v(t)$ in the interval $[a, b]$ i.e. $v(t) \in[a, b]$ where $0<a<b<\infty$. Without loss of generality we may assume that $x \in[0,1]$ and $t \in[0,1]$. We shall also assume that $v(\cdot)$ is a piecewise continuous function on $[0,1]$.

Put

$$
\bar{u}(x, t)=z(x, t)+\bar{u}_{0} \exp \left(-\frac{\beta}{v_{0}} x\right) .
$$

It is easy to demonstrate that (4.1)-(4.2) is equivalent to a system of the form

$$
\begin{gathered}
z_{x t}(x, t)+\beta \gamma z_{x}(x, t)+\frac{\beta}{v(t)} z_{t}(x, t)-\frac{\gamma \beta^{2} \bar{u}_{0}}{v_{0}} \exp \left(\frac{-\beta}{v_{0}} x\right)=0, \\
z(x, 0)=0, \quad z(0, t)=0 .
\end{gathered}
$$

The function $v=v(t)$ will be treated as a control in (4.3)-(4.4).

Suppose that each rapid or sudden change in the speed of the flow of the gas through the filter is expensive and should be taken into account in the general costs of air filtration. Consequently, the cost functional ought to have the form

$$
I(z, v)=\int_{0}^{1} \int_{0}^{1} f^{0}(x, t, z(x, t), v(t)) d x d t+\alpha N(v)+\beta S(v),
$$


where $\alpha, \beta>0, N(v)$ denotes the number of points of discontinuity of the control $v$,

$$
S(v)=\sup \left\{\frac{\left|v\left(t^{\prime}\right)-v(t)\right|}{\left|t^{\prime}-t\right|}, \quad t^{\prime} \neq t, t^{\prime}, t \in Q_{k}\right\},
$$

$Q_{k}, k=1,2, \ldots, q$, stand for the intervals of continuity of the control $v$.

Assume that the function $f^{0}$ satisfies (2.10). In that case, it can easily be noticed that control system (4.3)-(4.4) with cost functional (4.5) satisfies all the conditions of Theorem 3 (cf. Remark 1). Hence it appears that there exists some $v^{*}=v^{*}(t)$ which is the optimal speed of the flow of the gas through the filter.

The necessary optimality conditions which allows one to determine the optimal control $v^{*}$ will be considered in our next paper.

\section{Acknowledgement}

The paper was supported by KBN grant 211029101 .

\section{References}

[1] A. Bielecki, "Une remarque sur l'application de la méthode de Banach-Cocciopoli-Tichonov dans la théorie de l'équation $s=f(x, y, z, p, q)$ ", Bull. Acad. Pol. Sci. 4 (1956) 265-268.

[2] J. M. Blatt, "Optimal control with a cost of switching control", J. Austral. Math. Soc. Ser. B 19 (1976) 316-332.

[3] L. Cesari, Optimization - Theory and applications (Springer-Verlag, New York, 1983).

[4] K. Kibalczyc and S. Walczak, "Necessary optimality conditions for a problem with cost of rapid variation of control", J. Austral. Math. Soc. Ser. B 26 (1984) 45-55.

[5] M. A. Krasnosielski, Topological methods in the theory of nonlinear integral equations (Macmillan, New York, 1964).

[6] S. Lojasiewicz, An introduction to the theory of real functions (John Willey and Sons, Chichester, 1988).

[7] J. Matula, "On an extremum problem", J. Austral. Math. Soc. Ser. B 28 (1987) 376-392.

[8] E. S. Noussair, "On the existence of piecewise continuous optimal controls", J. Austral. Math. Soc. Ser. B 20 (1977) 31-37.

[9] S. Saks, Theory of the integral (Warsaw, 1937).

[10] D. E. Stewart, "A numerical algorithm for optimal control problems with switching costs", $J$. Austral. Math. Soc. Ser. B 34 (1992) 212-228.

[11] K. L. Teo and L. S. Jennings, "Optimal control with a cost on changing control", J. Optim. Theory Appl. 68 (2) (1991) 335-357.

[12] A. N. Tikhonov and A. A. Samarski, Equations of mathematical physics (Moscow, 1978).

[13] S. Walczak, "Absolutely continuous functions of several variables and their applications to differential equations", Bull. Polish Acad. Sci. Math. 35 (11-12) (1987) 733-744.

[14] S. Walczak, "On the differentiability of absolutely continuous functions of several variables", Bull. Polish Acad. Sci. Math. 36 (9-10) (1988) 513-520. 